\title{
Unilateral Combined Stereotactic Radiofrequency Pallidotomy And Thalamotomy For Idiopathic Parkinson's Disease'. A Prospective Observational Study With 24-Month Follow-Up
}

Mohamed Khaled Elkazaz ( $\square$ Mohamed.elkazaz@med.suez.edu.eg )

Suez Canal University https://orcid.org/0000-0002-7290-5744

Ali Salah Khedr

Suez Canal University

Maha Abd El Fattah

Suez Canal University

\section{Research}

Keywords: Parkinson's disease, Thalamotomy, Pallidotomy, Tremors, Spasticity, Stereotaxis, Radiofrequency

Posted Date: June 16th, 2021

DOI: https://doi.org/10.21203/rs.3.rs-525235/v1

License: (c) (i) This work is licensed under a Creative Commons Attribution 4.0 International License.

Read Full License 


\section{Abstract}

Study Design: Prospective study

Objective: To report efficacy of unilateral combined stereotactic radiofrequency pallidotomy and thalamotomy for Idiopathic Parkinson's disease.

Methods: Between June 2017 to December 2019, 62 patients with idiopathic Parkinson's disease underwent stereotactic radiofrequency pallidotomy and thalamotomy. Pre-operatively clinical assessment using the UPDRS and Hoen and Yahr scale for PD. Post-operatively clinical assessment using the UPDRS and Hoen and Yahr scale for PD, complications in 1,6,12 and 24 months.

Results: 60 patients completed two-year follow-up and fulfilled our criteria were recruited. The mean age was $57.47 \pm 9.90$. The average UPDRS off motor assessment results showed reduction after 1 month from 60.16 to 30.88 and at 24-month follow-up was 41.6. The average Yahr and Hoen scale 3.63 to 1.19 after 1-month and 24 months was 1.87. The average UPDRS constancy of tremors improved after 1-month from 3.53 to 0.75 . Improvement in constancy of tremors reached $75 \%$ of cases after 24 -month with average 1.62. the average UPDRS rigidity score improved at 1-month follow-up from 3.31 to 1.21. Total improvement of rigidity reached $63 \%$ after 24 -month. 2 patients had post-operative thalamic hematoma presented with hemiplegia, which was conservatively managed, and improved after 1-month with little deficit. 51.6\% had gait imbalance at 6-month follow-up. $22.5 \%$ showed dysarthria immediately while $12.9 \%$ totally resolved after 1-month follow-up. No recorded cases of infection, CSF leaks or cognitive dysfunction.

Conclusion: Our data suggest that Unilateral combined stereotactic radiofrequency pallidotomy and thalamotomy for Idiopathic PD is effective procedure.

\section{Introduction}

Lesioning surgeries in PD include target obliteration of a certain area in brain tissue in to disrupt maladaptive neuronal arrangements. An advancement in movement disorders when stereotactic procedures in lesioning were applied. Target selections were modulated various times until in 1960s when thalamotomy was used for tremors and pallidotomy was used for Bradykinesia and rigidity. $(1,2)$

Though lesioning procedure was executed for numerous years in particular patients with PD, their practice reduced in the 1960s following the presentation of levodopa. However, long term treatment with L-dopa has led to newly unfavorable side effects that refreshed the era of lesion surgeries.(3)

Lesioning procedures has since developed, and presently accessible methods include invasive procedures such as thermoablation, radiofrequency (RF) and laser interstitial thermal therapy (LITT). And less invasive ones such as MRI-guided high-intensity focused ultrasound (HIFU) thermal ablation (or MR- 
guided focused ultrasound, MRgFUS), stereotactic radiosurgery (SRS with the radiofrequency and thermaoablation are commonly used. $(4,5)$

In 1954, Hassler and Reichert (2) used thalamotomy to treat tremor associated with Parkinson's disease (PD). Ventrolateral thalamotomy has been considered to be the neurosurgical treatment of choice for disabling, drug-resistant parkinsonian and other types of tremor that tremor can be relieved not only by a thalamic lesion but also by a lesion in the posteroventral portion of the medial (internal) globus pallidum and ventralis intermedius (VIM) nucleus of the thalamus. Hassler and Reichert, In 1945, Proved that thalamotomy controls the tremors related to PD. Various studies had shown the role of VIM lesioning in control of PD medically refractory tremors or other types of tremors. (6-8).

One of the studies reviewed retrospectively the results in 60 patients with PD tremors, cerebellar tremors, essential tremor and post-traumatic tremor. These patients all had unilateral Vim thalamotomy. The mean follow-up of 53.4 months, PD tremors had marked improvement in $86 \%$ of the cases. essential tremor patients had similar improvement in $83 \%$ of cases. Results were not as favorable as PD tremors and essential tremors for those patients with post-traumatic tremor $(50 \%)$ or cerebellar tremor $(67 \%)$. Temporarily complications of thalamotomy were seen in $60 \%$ of cases includes dysarthria, dysphasia, confusion, dystonia, contralateral paresis or sensory disturbances. (9)

Lesion of the posteroventral segment of the internal globus pallidus (GPi) is efficient at treating contralateral tremor, rigidity, dyskinesia and bradykinesia. (10) Studies various studies proved this opinion. The first of these studies described an improvement in the off-state UPDRS motor score of $71 \%$ at 1 year following the surgery. (11) Lozano et al. (12)UPDRS motor score improved in the off-state by $30 \% 6$ months post operatively, although the UPDRS akinesia score improved only by $33 \%$. The gait score showed $15 \%$ improvement in the off-state and decrease in contralateral dyskinesias by $92 \%$. Baron et al. (13), described a $25 \%$ improvement in the motor score in the off-state by $25 \% 3$ months post operatively. Shannon et al. (14) described an improvement in $15 \%$ in the off-state motor score 6 months post operatively.

\section{Patients And Methods}

This prospective observational study for patients suffering from Idiopathic Parkinson's disease at Suez Canal University institution through June 2017 to January 2019. Total number of 62 patients underwent stereotactic unilateral combined pallidotomy "GPi" and Thalamotomy "Vim" using radiofrequency ablation for the management of Parkinson related tremors, bradykinesia and rigidity after careful counselling and acquiring consent from the patients. All patients have signed a consent for the publication purpose. This article followed the world medical association declaration of Helsinki ethical principles. Inclusion criteria included patients only diagnosed with idiopathic PD, with medication induced motor signs and refractory tremor and Hoehn and Yahr $>2$ stage off stage. Exclusion criteria included multiple comorbidities that increases morbidity and mortality risk of the surgery, Neuropsychiatric 
diseases, Dementia, postural instability and atypical PD "supranuclear gaze palsy, early postural instability or severe early dysautonomia". Failure to proceed to 24-month follow-up.

A multidisciplinary presurgical assessment is done by neurosurgeon and neurology team was a complete physical and mental assessment to the patients presented with PD. Neurology team was responsible for diagnosis confirmation and assessment of the medication trials the patients were offered. The off state was outlined as the state after withdrawal by 12 hours of anti PD treatment based in the Core assessment program for intracerebral transplantation committee definition (CAPIT). (15) UPDRS off state motor assessment was used in the following items in off state; medication induced dyskinesia pre-and post-operatively immediate, 6 months and 2 year.(16) Patients were also staged in Hoehn and Yahr scale in off state pre- and post-operatively immediate, 6 months and 1 year.(17) Preoperative brain imaging included an MRI and a CT brain one day prior to surgery.

\section{Operative details}

After admission patients are pre-operatively assessed by anesthesia team. Stereotactic system application "CRW" system is used under local anesthesia by scalp block. Patients then underwent CT brain with the frame and fiducials applied to it. Data is analyzed and target selection is done by waypoint navigator software FHC corporation selecting the preference points after fusion between preoperative MRI, CT brain and post fiducials CT brain as follows.

\section{Target selection}

Vim target from the posterior commissural point with $14 \mathrm{~mm}$ lateral, $6 \mathrm{~mm}$ anterior in the same AC-PC plane (Fig. 1) and GPi point from the mid commissural point is $21 \mathrm{~mm}$ lateral and $3 \mathrm{~mm}$ anterior to it at the MC plane (Fig. 1). Specific adjustments are done in relation to the lateral ventricular wall and internal capsule with the fusion images. Following, targets are transferred to Stereocheck ios by Moris (Fig. 2) application and target data acquisition is done in Entry, target $\mathrm{X}, \mathrm{Y}, \mathrm{Z}$. preoperative placement of the patient on the Mayfield. Patients is connected with neurophysiology monitoring for motor assessment. Sterile shaving and surgical draping is done. Data gained from Stereocheck is then transferred to phantom stereotaxis system and confirmed then the arc and ring is fixed upon patients' frame. Burr hole guided by trajectory from the software then durotomy.

\section{Electrode placement}

Insertion of the macroelectrode with bipolar 2-mm width, 3-mm length and 3-mm tip through a guiding tube with $2 \mathrm{~mm}$ diameter of the radiofrequency apparatus "Neuro N50 by Inomed" with impedance monitoring.

Functional localization is done by Macrostimulation with $2 \mathrm{~Hz}$ and $50 \mathrm{~Hz}$ in both points to assess proximity of the Vim target to VC and internal capsule and GPi target to its proximity to internal capsule. After confirmation of the target Vim underwent a temporarily lesioning " $50 \mathrm{C}$ for 50 seconds' followed by careful assessment of the patient if no deficit then followed by permanent lesion " $65 \mathrm{C}$ for 60 seconds" withdraw by $2-\mathrm{mm}$ and reapply permanent lesion. Gpi underwent a temporarily lesioning " $50 \mathrm{C}$ for 50 
seconds' followed by careful assessment of the patient then followed by permanent lesion " $72 \mathrm{C}$ for 60 seconds" withdrawal by 2-mm then reapply permanent lesion. Hemostasis and skin closure are done.

Post-operative assessment is done in 6-month and 2-year assessments for the UPDRS off state motor assessment and Hoehn and Yahr scale. Intra and Post-operative complications are reported.

Statistical analysis was done by SPSS IOS v26. paired t-test was implemented between means of variables.

\section{Results}

62 patients were enrolled in the study, 37 male and 25 females and the mean age is $57.47 \pm 9.90 .2$ patients failed to follow-up and excluded from the study.

The average UPDRS off motor assessment results showed marked reduction after 1 month from 60.16 to 30.88 (Table 1). At 6-month and 1-year follow-ups there was a non-significant rise in the average UPDRS off motor assessment to 39.1 and 42.3 respectively. At 24-month follow-up the average UPDRS off motor was 41.6. The average Yahr and Hoen scale 3.63 to 1.19 after 1-month follow-up (Table 1). At 6-month and 12-month follow-ups there was a non-significant rise to 1.5 and 1.9. 24 months post-operatively the average Yahr and Hoen scale was 1.87. (Graph 1 and 2)

Table 1

Clinical assessment pre-operative and the final follow-up (24-month) later

\begin{tabular}{|lllll|}
\hline Item & Pre-operative & 24-month & t-score & $p$-value \\
\hline UPDRS off state motor score & $60.16 \pm 3.8$ & $41.6 \pm 4.49$ & 10.23 & $<0.05^{\star}$ \\
\hline Yahr and Hoehn scale & $3.63 \pm 0.66$ & $1.87 \pm 0.61$ & 7.8 & $<0.05^{\star}$ \\
\hline $\begin{array}{l}\text { Results are in mean } \pm \text { SD. } \\
\text { * } \text {-value }<0.05 \text { is statistically significant at confidence interval 95\%. }\end{array}$ & \\
\hline
\end{tabular}

The average UPDRS constancy of tremors (Graph 3 ) were analyzed and showed a significant improvement after 1-month duration 3.53 to 0.75 . There was a non-significant rise in 6,12 to 1.23 and 1.72 respectively. Total improvement in constancy of tremors reached up to $75 \%$ of cases after 24 -month with average 1.62

In addition, the average UPDRS rigidity score (Graph 4) also showed significant improvement at 1-month follow-up from 3.31 to 1.21. There was a non-significant rise in 6,12 to 1.9 and 2.3. At 24-month follow-up the average reached 2.1. Total improvement of rigidity reached up to $63 \%$ of cases after 24 -month.

2 patients (3.2\%) had thalamic hematoma that was discovered post-operatively with hemiplegia (Fig. 3) which was conservatively managed, and patients improved after 1-month with little residual deficit. Also noticed in the study that 32 patients $(51.6 \%)$ had gait imbalance noticed at 6-month follow-up with leaning towards the contralateral side to the lesioning. 14 patients $(22.5 \%)$ of cases showed dysarthria 
immediately post-operative where 8 patients $(12.9 \%)$ totally resolved after 1-month follow-up. No recorded cases of infection, CSF leaks or cognitive dysfunction.

\section{Discussion}

Spiegel et al. in 1947 introduced the era of brain stereotactic surgery.(18) Later it became an evolution in this field in the management of PD. Hassler successfully managed to control PD symptoms by thalamotomy.(19) Traditionally, thalamotomy alone was used as a maneuver for PD. But after Leksell's evolutionary introduction to the Gpi lesioning rigidity and bradykinesia were managed along with tremors. (6) Long term studies on isolated thalamotomies had shown that patients are still disabled with rigidity. In addition studies which favored isolated lesion in GPi can control both rigidity and tremors that was found later to be insufficnet to control the tremors. $(20,21)$ Vim lesioning aims to disrupt the rubrothalamocortical circuit controlling abnormal impulses initiating tremors.(22, 23) while GPi lesioning disconnect the inhibitory outflow of the Gpi to the VOa nucleus and the PPN. $(22,24)$

In this series we had a success rate in improving patients UPDRS and experiencing less relapse and complications. Marcelo et al. reported improvement of thalamotomy procedure in suppressing tremors in PD with $75 \%$ in upper limb and $73 \%$ in lower limb tremors.(25)

Fayed et al. reported successful results in combined pallidotomy and thalamotomy over pallidotomy alone in improving patients functional state and controlling PD.(26) Several mechanisms were postulated regarding the persistence of tremors after lesioning the Gpi alone as the Gpi lesion does not directly disconnect the rubrothalamocortical loop as it may exacerbate by the disinhibition of the reticulospineal system. $(23,27)$ Locano et al. stated that combined Vim and GPi lesioning can treat broad range of symptoms in PD immediately with no or little risk on the patients.(22)

\section{Conclusion}

Unilateral combined Vim/Gpi had shown to be very effective in controlling PD symptoms and improve the overall functional state of the patients. Despite a successful technique, few studies were involved in it. It showed to be potent and carries little risk for the patients. We advocate that case-control studies should be directed to assess this technique.

\section{Limitations}

The small sample size affected the statistical testing of the results.

\section{Abbreviations}

CAPIT: Core assessment program for intracerebral transplantation committee

CRW: Cosman Roberts Wells 
CSF: cerebrospinal fluid

CT: Computed tomography

GPi: Globus pallidus internus

HIFU: High-intensity focused ultrasound

LITT: laser interstitial thermal therapy

MRgFUS: Magnetic resonance -guided focused ultrasound

MRI: Magnetic resonance imaging

PD: Parkinson's Disease

RF: Radiofrequency

UPDRS: Unified Parkinson's disease rating scale

Vim: Ventralis intermedius

\section{Declarations}

\section{Ethics approval and consent to participate}

This research was revised and accepted by the research committee in Suez Canal University hospitals and accepted. Patients were consented in written format before entering the study for undergoing the procedure and publication purposes.

\section{Consent for Publications}

Patients were consented for the publication purpose of their medical data.

Availability of data and materials

The datasets used in this research are available in the neurosurgical department, Suez Canal University and are available upon requested

Competing interests

The authors declare that they have no competing interests.

\section{Funding}

There is no funding for this research 
M.E, Investigation and Formal analysis of the data and writing original draft.

A.K Surgical planning and execution, Methodology, Resources, Validation, writing review and editing, supervision and Project administration

M.A Writing review and editing and planning Physiotherapeutic plan for the patients.

\section{Acknowledgments}

Special thanks to the Neurosurgery department in Suez Canal University for the continuous support.

\section{References}

1. Svennilson E, Torvik A, Lowe R, Leksell L. Treatment of parkinsonism by stereotactic thermolesions in the pallidal region. A clinical evaluation of 81 cases. Acta Psychiatr Scand. 1960;35(3):358-77.

2. Hassler R, Riechert T. Indications and localization of stereotactic brain operations. Nervenarzt. 1954;25(11):441.

3. Marsden CD, Parkes JD. Success and problems of long-term levodopa therapy in Parkinson's disease. Lancet. 1977;309(8007):345-9.

4. Walters $\mathrm{H}$, Shah BB. Focused ultrasound and other lesioning therapies in movement disorders. Curr Neurol Neurosci Rep. 2019;19(9):66.

5. Jourdain VA, Schechtmann G. Health economics and surgical treatment for Parkinson's disease in a world perspective: results from an international survey. Stereotact Funct Neurosurg. 2014;92(2):719.

6. Laitinen LV, Bergenheim AT, Hariz MI. Leksell's posteroventral pallidotomy in the treatment of Parkinson's disease. J Neurosurg. 1992;76(1):53-61.

7. Nagaseki $Y$, Shibazaki T, Hirai T, Kawashima $Y$, Hirato $M$, Wada $H$, et al. Long-term follow-up results of selective VIM-thalamotomy. J Neurosurg. 1986;65(3):296-302.

8. Fox MW, Ahlskog JE, Kelly PJ. Stereotactic ventrolateralis thalamotomy for medically refractory tremor in post-levodopa era Parkinson's disease patients. J Neurosurg. 1991;75(5):723-30.

9. Jankovic J, Cardoso F, Grossman RG, Hamilton WJ. Outcome after stereotactic thalamotomy for parkinsonian, essential, and other types of tremor. Neurosurgery. 1995;37(4):680-7.

10. Lai EC, Jankovic J, Krauss JK, Ondo WG, Grossman RG. Long-term efficacy of posteroventral pallidotomy in the treatment of Parkinson's disease. Neurology. 2000;55(8):1218-22.

11. Dogali M, Fazzini E, Kolodny E, Eidelberg D, Sterio D, Devinsky O, et al. Stereotactic ventral pallidotomy for Parkinson's disease. Neurology. 1995;45(4):753-61.

12. Lozano AM, Lang AE, Galvez-Jimenez N, Miyasaki J, Duff J, Hutchison WD, et al. Effect of GPi pallidotomy on motor function in Parkinson's disease. Lancet. 1995;346(8987):1383-7. 
13. Baron MS, Vitek JL, Green J, Kaneoke Y, Hashimoto T, Turner RS, et al. Treatment of advanced Parkinson's disease by posterior GPi pallidotomy: 1-year results of a pilot study. Ann Neurol Off J Am Neurol Assoc Child Neurol Soc. 1996;40(3):355-66.

14. Shannon KM, Penn RD, Kroin JS, Adler CH, Janko KA, York M, et al. Stereotactic pallidotomy for the treatment of Parkinson's disease: Efficacy and adverse effects at 6 months in 26 patients. Neurology. 1998;50(2):434-8.

15. Langston JW, Widner H, Goetz CG, Brooks D, Fahn S, Freeman T, et al. Core assessment program for intracerebral transplantations (CAPIT). Mov Disord Off J Mov Disord Soc. 1992;7(1):2-13.

16. Disease, MDSTF on RS for P. The unified Parkinson's disease rating scale (UPDRS): status and recommendations. Mov Disord. 2003;18(7):738-50.

17. Hoehn MM, Yahr MD. Parkinsonism: onset, progression, and mortality. Neurology. 1998;50(2):318.

18. Spiegel EA, Wycis HT, Marks M, Lee AJ. Stereotaxic apparatus for operations on the human brain. Science. 1947;106(2754):349-50.

19. Hassler R. Die extrapyramidalen Rindensysteme und die zentrale Regelung der Motorik. Dtsch Z Nervenheilkd. 1956;175(3):233-58.

20. Okun MS, Vitek JL. Lesion therapy for Parkinson's disease and other movement disorders: update and controversies. Mov Disord Off J Mov Disord Soc. 2004;19(4):375-89.

21. Laitinen LV, Bergenheim AT, Hariz MI. Ventroposterolateral pallidotomy can abolish all parkinsonian symptoms. Stereotact Funct Neurosurg. 1992;58(1-4):14-21.

22. Iacono RP, Henderson JM, Lonser RR. Combined stereotactic thalamotomy and posteroventral pallidotomy for Parkinson's disease. J Image Guid Surg. 1995;1(3):133-40.

23. Narabayashi $\mathrm{H}$. Neurophysiological ideas on pallidotomy and ventrolateral thalamotomy for hyperkinesis. Stereotact Funct Neurosurg. 1962;22(3-5):291-303.

24. Ohye C. Depth microelectrode studies. Stereotaxy Hum brain. 1982;372-89.

25. Linhares MN, Tasker RR. Microelectrode-guided thalamotomy for Parkinson's disease. Neurosurgery. 2000;46(2):390-8.

26. Fayed ZY, Radwan H, Aziz M, Eid M, Mansour AH, Nosseir M, et al. Combined Unilateral Posteroventral Pallidotomy and Ventral Intermediate Nucleus Thalamotomy in Tremor-Dominant Parkinson's Disease versus Posteroventral Pallidotomy Alone: A Prospective Comparative Study. Stereotact Funct Neurosurg. 2018;96(4):264-9.

27. Sandyk R, lacono RP. The relationship of the reticular system to the primary pathoetiology of Parkinson's disease. Int J Neurosci. 1988;42(3-4):297-300.

\section{Figures}



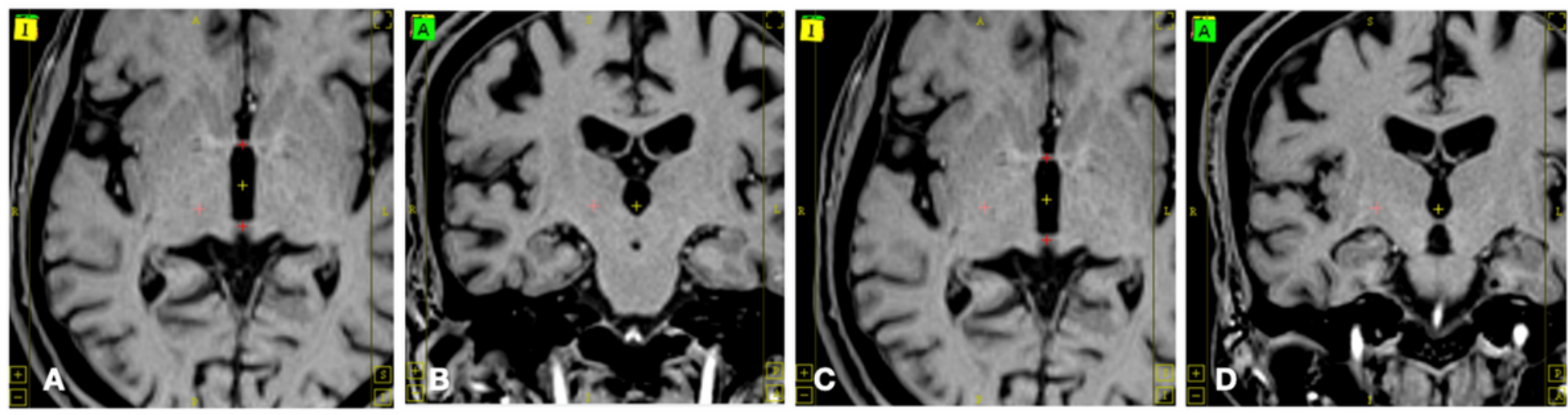

Figure 1

Case presentation 56 male patient with PD planning for right unilateral combined Vim and Gpi lesioning. A, axial MRI brain T1W with Vim target "red crosshair right to third ventricle". B, Coronal MRI brain T1W with Vim target "red crosshair right to third ventricle". C, axial MRI brain T1W with Gpi target "red crosshair right to third ventricle". D, Coronal MRI brain T1W with Gpi target "red crosshair right to third ventricle".

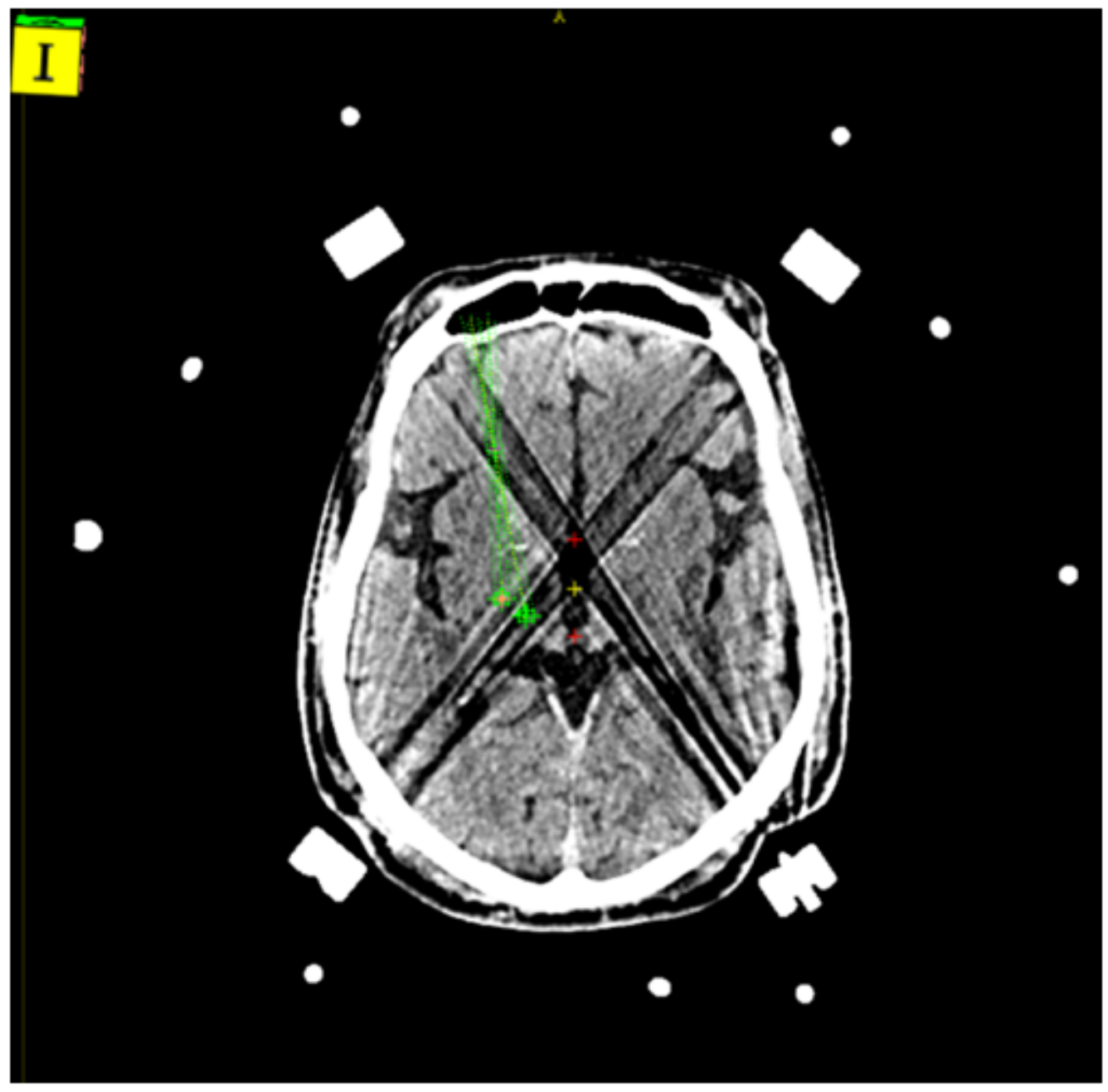

Figure 2 
Axial CT brain with ring and fiducial marks for planning in Stereocheck ios by Moris.
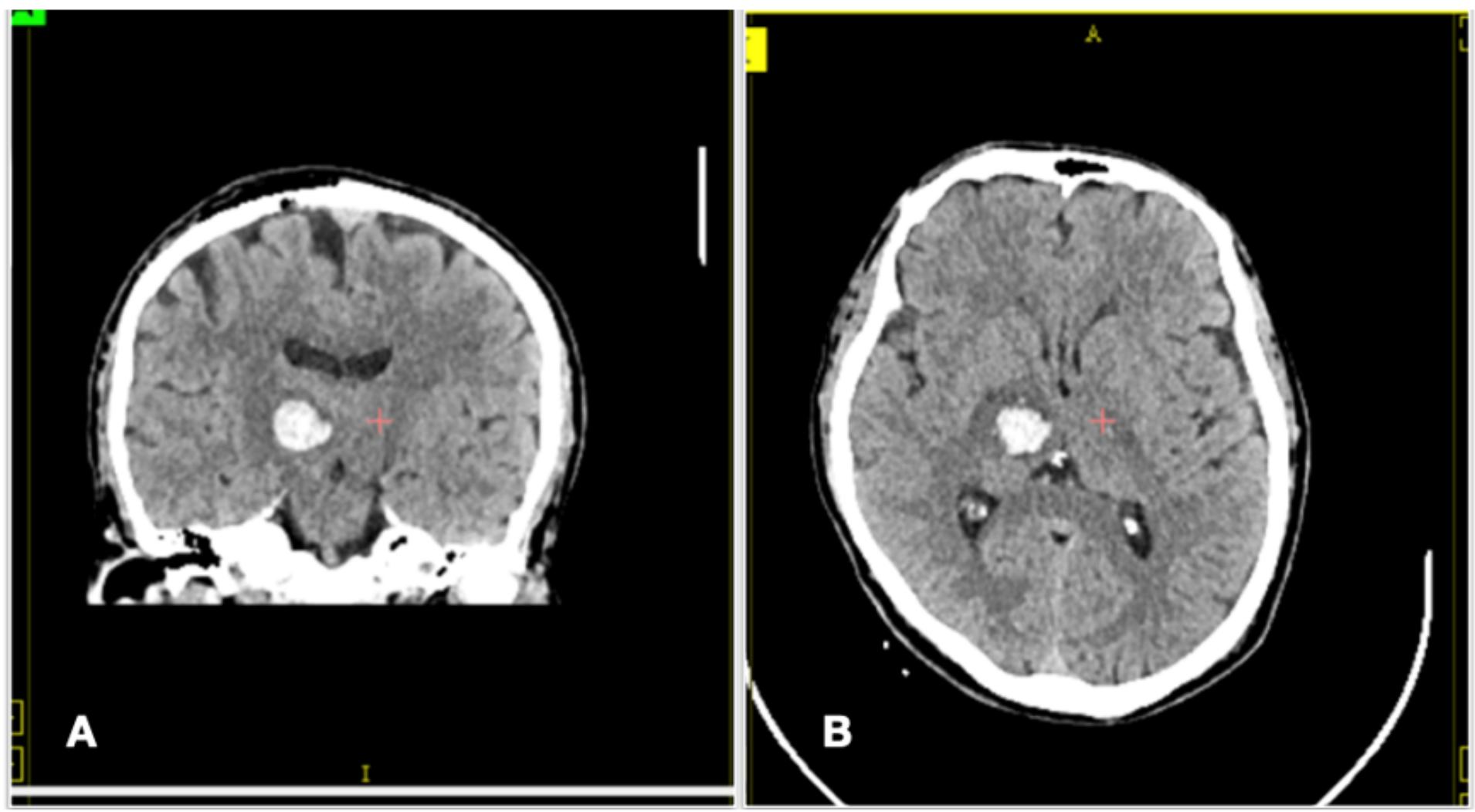

Figure 3

A, Coronal CT brain with thalamic hematoma post-lesioning. B, Axial CT 


\section{UPDRS off motor state}

70

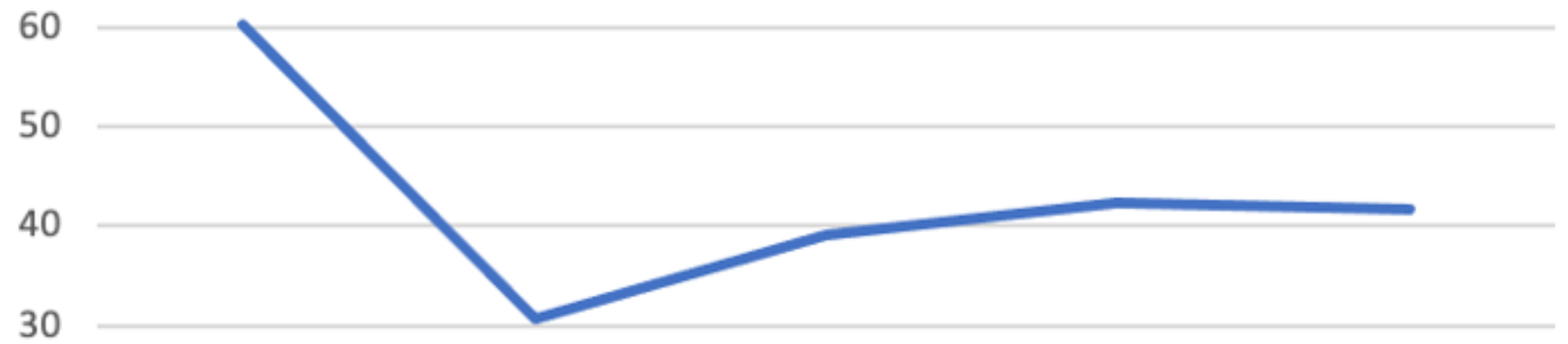

20

10

0

Pre-operative 1-month 6-month 12-month 24-month

Figure 4

Graph1: UPDRS off motor state assessment. 


\section{Yahr and Hoen scale}

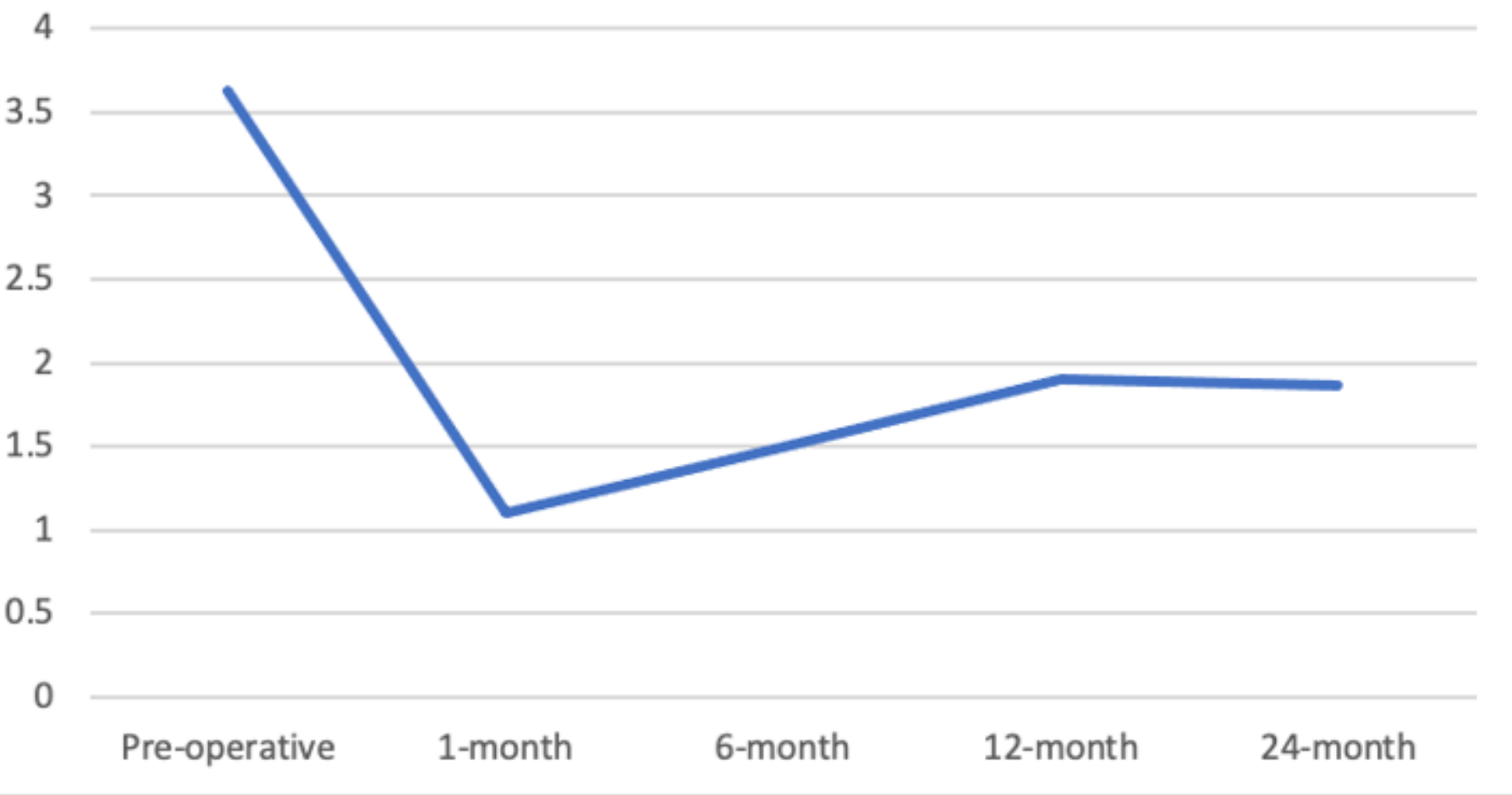

Figure 5

Graph2: Yahr and Hoen assessment 


\section{UPDRS constancy of tremors}

4

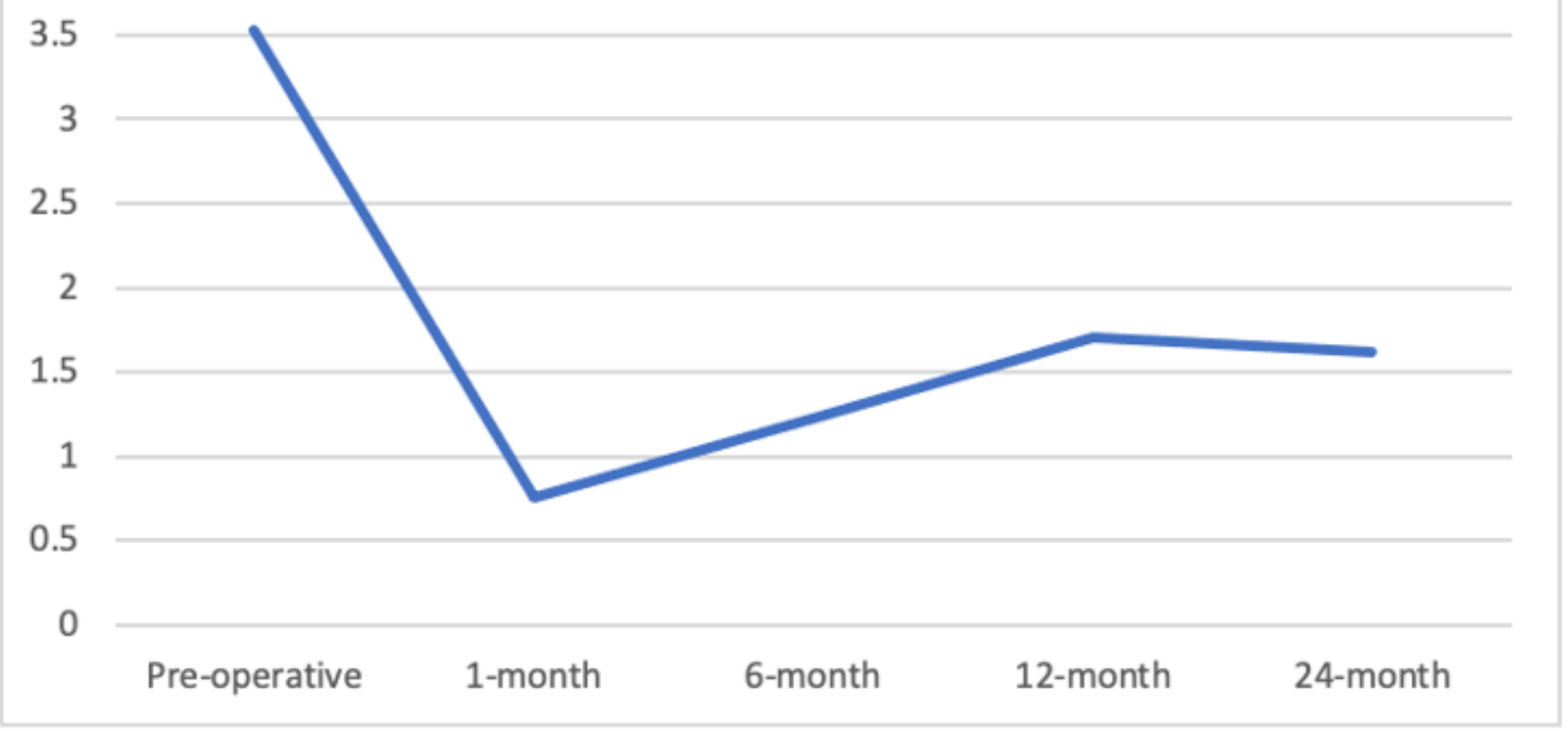

Figure 6

Graph3: UPDRS constancy of rest tremors assessment 


\section{UPDRS Rigidity}

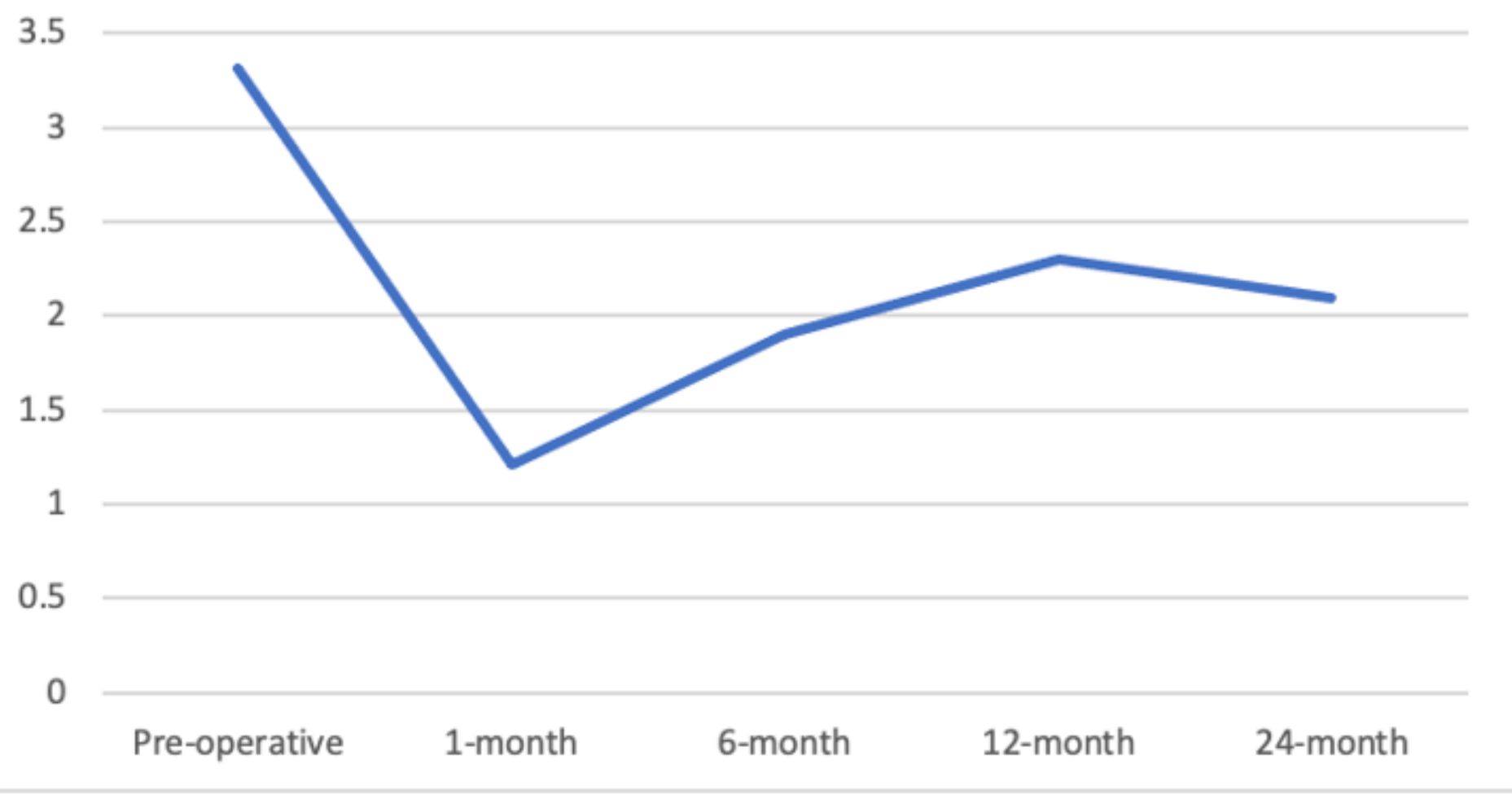

Figure 7

Graph4: UPDRS rigidity assessment 